\title{
Superimposing positive end-expiratory pressure during partial liquid ventilation in experimental lung injury
}

\author{
U. Kaisers*, R. Kuhlen*, U. Keske**, A. Sommerer*, A. Mohnhaupt+, \\ K.J. Falke*, R. Rossaint*
}

Superimposing positive end-expiratory pressure during partial liquid ventilation in experimental lung injury. U. Kaisers, R. Kuhlen, U. Keske, A. Sommerer, A. Mohnhaupt, K.J. Falke, R. Rossaint. @ $\mathrm{ERS}$ Journals Ltd 1998.

ABSTRACT This study was undertaken to determine the effects of superimposing incremental levels of positive end-expiratory pressure (PEEP) during partial liquid ventilation (PLV) on gas exchange, respiratory mechanics and morphological changes in experimental acute lung injury (ALI).

In a prospective trial, six pigs weighing $30 \pm 5 \mathrm{~kg}($ mean $\pm \mathrm{sD})$ were tracheotomized, submitted to pressure-controlled mechanical ventilation (pc-CMV) and depleted of surfactant by lung lavage. Animals were then mechanically ventilated with three levels of PEEP: 0.5, 1.0 and 1.5 kPa. PLV was then initiated by intratracheal instillation of $30 \mathrm{~mL} \cdot \mathrm{kg}^{-1}$ perfluorocarbon, followed by pc-CMV with PEEP $0.5,1.0$ and $1.5 \mathrm{kPa}$. Computed tomography (CT)-based analyses of lung volumes and density were obtained after lung lavage, in PLV and during the combined application of PLV and PEEP. Simultaneously, haemodynamics, gas exchange, dynamic compliance $(C \mathrm{dyn})$ and dynamic resistance $(R d y n)$ were determined. Statistical analysis was performed using multivariate analyses of variance for repeated measures $(\mathbf{p}<0.05)$.

In ALI and before PLV, the application of PEEP significantly reduced cardiac output and intrapulmonary shunt. Arterial oxygen tension $\left(\mathrm{Pa}_{\mathrm{a}}, \mathrm{O}_{2}\right)$ was increased from 6.9 $\mathrm{kPa}(52(42,54) \mathrm{mmHg})$ (median, (25th and 75 th percentile)) to $8.6 \mathrm{kPa}(65(52,133)$ $\mathrm{mmHg}$ (PEEP 1.0 kPa) and $15.6 \mathrm{kPa}(117(90,195) \mathrm{mmHg})(\mathrm{PEEP} 1.5 \mathrm{kPa})(\mathrm{p}<0.05)$. The lung volume obtained by $\mathrm{CT}$ increased, $\mathrm{CT}$ density was reduced $(\mathrm{p}<0.05), C \mathrm{dyn}$ tended to increase and Rdyn to decrease (nonsignificant). PLV increased arterial carbon dioxide tension and reduced $\mathrm{pH}(\mathrm{p}<0.05)$. CT lung volume and lung density were increased $(\mathrm{p}<0.05)$. Superimposing PEEP on PLV increased $P \mathrm{a}_{2}, \mathrm{O}_{2}$ from $9.3 \mathrm{kPa}(70$ $(52,124) \mathrm{mmHg}$ (PEEP 0.5 kPa) to $12.9 \mathrm{kPa}(97(55,233) \mathrm{mmHg})(P E E P 1.0 \mathrm{kPa})$ and $40.3 \mathrm{kPa}(303(64,426) \mathrm{mmHg})($ PEEP $1.5 \mathrm{kPa})(\mathrm{p}<0.05)$, but had no significant effect on CT lung volume and density.

It was concluded that in experimental lung injury, positive end-expiratory pressure provided alveolar recruitment. The combined application of positive end-expiratory pressure and partial liquid ventilation significantly augmented oxygenation and might eventually allow either a reduction in the volumes of perfluorocarbons required, or a reduction in positive end-expiratory pressure necessary to maintain pulmonary gas exchange in acute lung injury.

Eur Respir J 1998; 11: 1035-1042.
*Klinik für Anästhesiologie und Operative Intensivmedizin, **Strahlenklinik und Poliklinik, and +Forschungs-EDV und Statistik, Virchow-Klinikum, Medizinische Fa-kultät der Humboldt Universität zu Berlin, Germany.

Correspondence: U. Kaisers

Klinik für Anästhesiologie und Operative Intensivmedizin

Virchow-Klinikum der Humboldt Universität zu Berlin

Augustenburger Platz 1

13353 Berlin

Germany

Fax: 493045051900

Keywords: Acute lung injury acute respiratory distress syndrome computed tomographic image partial liquid ventilation perfluorocarbons

positive end-expiratory pressure-ventilation

Received: June 231997

Accepted after revision January 161998

Supported by DFG (RO 2000-1/1)
In acute lung injury (ALI), mechanical ventilation with positive end-expiratory pressure (PEEP) is a mainstay in therapy, preventing alveolar end-expiratory collapse, and thus increasing end-expiratory lung volumes and functional residual capacity (FRC) [1]. Recent studies have demonstrated that in acute respiratory distress syndrome (ARDS) airway collapse concentrates in dependent lung regions owing to the increased weight of the infiltrated lung. The recruiting effects of PEEP are therefore mainly due to a counterforce mechanism preventing compression atelectasis [2].

Partial liquid ventilation (PLV), first described by FuHRMAN et al. [3], is a novel technique to assist mechanical ventilation, in which the lungs are filled with perfluorocarbons (PFC) to a volume approximating the
FRC or smal-ler and then gas ventilated using a standard mechanical ventilator.

PFC are radiopaque, dense liquids with high spreading coefficients and an excellent capacity for carrying oxygen and carbon dioxide. Intrapulmonary distribution of PFC is inhomogeneous and likely to follow the gravitational gradient, preferentially pooling in dependent lung areas. The beneficial effects of PLV on gas exchange are partly attributed to the filling and distending of dependent alveoli with the noncompressible PFC, thereby reopening air spaces and preventing end-expiratory collapse. In ALI, PLV might thus provide a vertically graded PEEP-like effect [4]. Consequently, it has been suggested that during PLV, additional recruitment of alveoli by PEEP might be of less importance [5]. 
Despite an extensive number of studies on PLV, the issue of characterizing appropriate PEEP levels during PLV has not been systematically evaluated. Moreover, in ALI, the in vivo intrapulmonary distribution of PFC during PLV combined with PEEP has to be established.

This study was undertaken to clarify the physiological and morphological response to the combined application of PLV and incremental levels of PEEP in an animal model of ARDS.

\section{Material and methods}

\section{Animal preparation}

Six female pigs (Deutsches Hausschwein) with a body weight of $30 \pm 5 \mathrm{~kg}$ were included in the study. The experimental protocol was approved by the local government and the experiments were performed in accordance with the Helsinki convention for the use and care of animals.

Animals were included in the study only when no signs of infection were perceptible prior to the experiment. Premedication was achieved with ketamine $\left(10 \mathrm{mg} \cdot \mathrm{kg}^{-1}\right.$ i.m. $)$, midazolam $\left(0.5 \mathrm{mg} \cdot \mathrm{kg}^{-1}\right.$ i.m. $)$ and atropine $\left(0.01 \mathrm{mg} \cdot \mathrm{kg}^{-1}\right.$ i.m.). After peripheral venous access, anaesthesia was induced with i.v. methohexital $\left(8 \mathrm{mg} \cdot \mathrm{kg}^{-1}\right)$ and fentanyl $(0.01$ $\left.\mathrm{mg} \cdot \mathrm{kg}^{-1}\right)$ and maintained with a continuous infusion of methohexital $\left(50 \mu \mathrm{g} \cdot \mathrm{kg}^{-1} \cdot \mathrm{min}^{-1}\right)$ and fentanyl $\left(3 \mu \mathrm{g} \cdot \mathrm{kg}^{-1} \cdot \mathrm{min}^{-1}\right)$. All animals received muscle relaxation by an initial bolus of pancuronium bromide $\left(0.15 \mathrm{mg} \cdot \mathrm{kg}^{-1}\right)$ followed by a continuous infusion $\left(2.5 \mu \mathrm{g} \cdot \mathrm{kg}^{-1} \cdot \mathrm{min}^{-1}\right)$ throughout the experiment. Immediately after induction, the pigs were tracheotomized and intubated with a 7.5-8.0 mm endotracheal tube, fitted with a heat moisture exchanger with bacterial filter.

Animals were then submitted to pressure-controlled mechanical ventilation (pc-CMV) (Servo 900C; Siemens Elema, Lund, Sweden) limiting peak inspiratory pressure to $ð 40 \mathrm{kPa}$, at a frequency of $18 \cdot \mathrm{min}^{-1}$, with an inspiratory oxygen fraction $\left(F \mathrm{I}, \mathrm{O}_{2}\right)$ of 1.0, an inspiratory/expiratory ratio of $1: 1$ and a PEEP of $0.5 \mathrm{kPa}$. The inspiratory pressure support and the respiratory rate were adjusted after obtaining two arterial blood gas analyses separated by 20 min in order to keep the animals normocapnic (arterial carbon dioxide tension $\left.\left(P \mathrm{a}, \mathrm{CO}_{2}\right)=5.3 \pm 0.8 \mathrm{kPa}(40 \pm 6 \mathrm{mmHg})\right)$. Temperature was maintained within $\pm 0.5^{\circ} \mathrm{C}$ of the prestudy range using a heating pad, and a continuous infusion of balanced electrolyte solution $\left(5 \mathrm{~mL} \cdot \mathrm{kg}^{-1} \cdot \mathrm{h}^{-1}\right)$ was given during the study. During the study, no cardiotonic or vasoactive drugs were administered.

Arterial and central venous access were performed percutaneously. The pulmonary artery catheter (model 93A431-7.5 F; Baxter Healthcare Corporation, Irvine, CA, USA) was advanced from the femoral vein using a $8.5 \mathrm{Fr}$ sheath (Baxter). An $18 \mathrm{G}$ arterial line (Vygon, Ecouen, France) was introduced into the femoral artery. The arterial line and the pulmonary artery catheter were used for blood sampling and the collection of haemodynamic data. Cardiac frequency was determined from the blood pressure curve. Central venous pressure (CVP), mean arterial pressure (MAP), mean pulmonary artery pressure (PAP) and pulmonary artery wedge pressure (PCWP) were assessed using a disposable transducer (Baxter) and a moni- toring system (Model 66 S; Hewlett-Packard; Böblingen, Germany). Measurements were taken in the supine position with zero reference level at the midaxilla, and vascular pressures were the average taken at end-expiration from three successive respiratory cycles. Cardiac output (CO) was determined with standard thermodilution techniques (Baxter) and expressed as the mean of four measurements using injections of saline $\left(10 \mathrm{~mL}\right.$ at $\left.1-5^{\circ} \mathrm{C}\right)$ arbitrarily performed during different phases of the respiratory cycle.

All blood samples (arterial and mixed venous) were collected anaerobically, placed in ice and analysed within 5 min using standard blood-gas electrodes (ABL 520; Radiometer, Copenhagen, Denmark), and spectrophotometry (OSM 3 Hemoximeter; Radiometer) was performed to obtain the total haemoglobin concentration, arterial oxygen saturation $\left(\mathrm{Sa}_{\mathrm{a}} \mathrm{O}_{2}\right)$ and mixed venous oxygen saturation $\left(\mathrm{Sv}, \mathrm{O}_{2}\right)$.

The following calculations were made. Arterial and mixed venous oxygen content $\left(\mathrm{mL} \cdot \mathrm{dL}^{-1}\right)$ were calculated from the following:

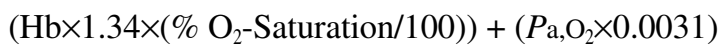

where $\mathrm{Hb}$ denotes haemoglobin and arterial oxygen tension $\left(\mathrm{Pa}, \mathrm{O}_{2}\right)$. The arteriovenous oxygen content difference $\left.(\mathrm{AvDO})_{2}\right)$ was calculated from: arterial oxygen content $\left(C \mathrm{a}, \mathrm{O}_{2}\right)$ - mixed venous oxygen content $\left(\mathrm{C}_{\mathrm{v}}, \mathrm{O}_{2}\right)$. The pulmonary right-to-left shunt $\left(Q_{\mathrm{s}} / Q \mathrm{t}\right)$ was calculated from:

$$
\left(C \mathrm{c}, \mathrm{O}_{2}-C \mathrm{a}, \mathrm{O}_{2}\right) /\left(C \mathrm{c}, \mathrm{O}_{2}-C \mathrm{v}, \mathrm{O}_{2}\right)
$$

where $C \mathrm{c}, \mathrm{O}_{2}$ denotes pulmonary end-capillary oxygen content, with the assumption that end-capillary $\mathrm{PO}_{2}$ is equivalent to alveolar $\mathrm{PO}_{2}\left(P \mathrm{~A}, \mathrm{O}_{2}\right)$ ("alveolar" during PLV refers to the gas adjacent to the fluid layer according to the suggestions of MATES et al. [6]). During PLV, $P A, O_{2}$ (at an $F \mathrm{I}_{2} \mathrm{O}_{2}$ of 1.0) was estimated as: Barometric pressure - $P \mathrm{~A}, \mathrm{CO}_{2}-$ water vapour pressure $\left(\mathrm{PH}_{2} \mathrm{O}\right)$ - $\mathrm{PFC}$ vapour pressure (PPFC) (8.1 kPa (61 mmHg) for FC 3280).

Arterial oxygen delivery $\left(\mathrm{DO}_{2}\right)\left(\mathrm{mL} \cdot \mathrm{min}^{-1}\right)=$ cardiac output $\times$ arterial oxygen content $\times 10$

Oxygen uptake $\left(\mathrm{VO}_{2}\right)\left(\mathrm{mL} \cdot \mathrm{min}^{-1}\right)=$ cardiac output $\times$ (arterial oxygen content mixed venous oxygen content $) \times 10$

\section{Flow and volume data}

Measurements of airflow $(V)$ were performed using a heated Fleisch No. 2 pneumotachograph (Metabo, Epalinges, Switzerland) placed between the y-piece of the respiratory tubing and the endotracheal tube (ETT), connected to a differential pressure transducer (Fumes Controls, Bexhill, UK). The pneumotachograph was calibrated before each experiment. Airway pressure $(P$ aw $)$ was measured with a probe placed at the outer end of the ETT and connected to a pressure transducer (Sensym, Milpitas, USA) via a $20 \mathrm{~cm}$ sampling line. Both $P$ aw and $V$ were sampled with a frequency of $100 \mathrm{~Hz}$ converted by an analogue-digital converter and stored on a personal computer. The pressure and flow waves and the pressure-volume (PV) loops obtained from the integrated flow signal were displayed on a computer screen. 
Respiratory mechanics were determined using a leastsquare fitting method for selected parts of the PV loop according to the method of GuTTMANN, described in detail elsewhere [7]. Since the ETT imposes a significant additional resistance on the respiratory system, the pressure drop across the ETT (PETT) was also calculated according to the method described by GutTmann [8]. Using this method, tracheal pressure $(P$ trach) was calculated as $P$ trach $=P$ aw $-P$ ETT . For all calculations of respiratory mechanics, the calculated $P$ trach was used, thereby eliminating the pressure drop due to the imposed resistance of the ETT. At the end of each step of the protocol, respiratory mechanics data of at least 10 consecutive breaths were averaged and stored for analyses.

\section{Induction of acute lung injury}

The lavage procedure was performed according to the method of surfactant depletion described by Lachmann and co-workers [9]. After disconnection from the ventilator, the animal was positioned in the supine, reverse Trendelenburg position at an angle of $60-70^{\circ}$ and the lungs were completely filled with warmed saline $\left(0.15 \mathrm{M}, 37^{\circ} \mathrm{C}, 40\right.$ $\mathrm{mL} \cdot \mathrm{kg}^{-1}$ ) via the ETT. The animal was then tilted $45^{\circ}$ with the head down and the lavage fluid was drained by gravitational force until mean arterial pressure markedly decreased and mean pulmonary artery pressure increased. After each lavage, the animals were reconnected to the ventilator for at least $20 \mathrm{~min}$ before the next lavage was performed. A minimum of four lavages was performed in the supine position, followed by three to four lavages in the prone position. Beginning with the third lavage onwards, arterial blood gas samples were taken at each lavage and the ventilator settings were adapted in order to achieve a $\mathrm{pH}>7.20$. Subsequent arterial blood gas samples and haemodynamic measurements were taken every $15 \mathrm{~min}$. An increase in $\mathrm{Pa}, \mathrm{O}_{2}$ within 60 min resulted in repetitive lavages until arterial $P \mathrm{a}, \mathrm{O}_{2}$ was persistently $<13.3$ $\mathrm{kPa}(100 \mathrm{mmHg})$, considered to indicate severe ALI.

\section{Experimental protocol}

The PFC used in this study (FC 3280; 3M Chemical Products, Neuss, Germany) is a highly purified radiopaque $\mathrm{C}_{8} \mathrm{~F}_{18}$ molecule. At $25^{\circ} \mathrm{C}$, FC 3280 has a density of $1.75 \mathrm{~g} \cdot \mathrm{cm}^{-3}$, a viscosity of 0.7 centistokes, a vapour pressure of $8.1 \mathrm{kPa}(61 \mathrm{mmHg})$ and a surface tension of 12 $\mathrm{mN} \cdot \mathrm{m}^{-1}$. It dissolves $40 \mathrm{~mL} \mathrm{O}$ and $192 \mathrm{~mL} \mathrm{CO}_{2}$ per 100 $\mathrm{mL}$ of PFC (3M product data).

After induction of ALI, animals were transferred to the computed tomography (CT) suite using a transportable ventilator (Servo 900C) and monitoring system (Hewlett Packard, 66S). The following experimental protocol was performed at the CT suite. After recording initial CT scans representing ALI and simultaneously measuring haemodynamics, gas exchange and lung mechanics during pc-CMV with different levels of PEEP $(0.5,1.0$ and $1.5 \mathrm{kPa}), 30 \mathrm{~mL} \cdot \mathrm{kg}^{-1}$ of FC 3280 was instilled into the trachea with the animal in the supine position during inspiration using a swivel-connector (Portex, Kent, UK). $\mathrm{Pc}-\mathrm{CMV}$ was resumed thereafter without changes in ven- tilator settings except for PEEP. After instillation, CT scans of the lungs, gas exchange, haemodynamics and lung mechanics were obtained during pc-CMV at three different levels of PEEP: $0.5,1.0$ and $1.5 \mathrm{kPa}$. Each step of data acquisition at each level of PEEP lasted for $10 \mathrm{~min}$, so that the entire measurements lasted for approximately $60 \mathrm{~min}$.

\section{Computed tomography protocol}

CT of the lungs was performed with a Somatom plus CT scanner (Siemens, Erlangen, Germany). The scans were performed in a spiral CT technique at $165 \mathrm{~mA}$ and $137 \mathrm{kV}$ with a slice thickness of $10 \mathrm{~mm}$. The system enables 24 rotations at 1 rotation. $\mathrm{s}^{-1}$. Table feed was $10 \mathrm{~mm}$. Thus, whenever the craniocaudal diameter of the lungs exceeded $23 \mathrm{~cm}$, a second spiral CT was performed to cover the entire lung. Spiral CT scans of the whole lungs were performed at the following respirator settings: fixed expiration ("expiratory hold" on Servo 900C) at PEEP settings of $0.5,1.0$, and $1.5 \mathrm{kPa}$. An initial CT scan series was performed immediately before the administration of PFC (following ALI) and scans were repeated immediately after administration of $30 \mathrm{~mL} \cdot \mathrm{kg}^{-1} \mathrm{PFC}$. Owing to lung lavage-induced pulmonary oedema and the significant amounts of PFC administered, automatic contour-finding programmes could not be implemented to calculate lung volume. In the CT scans acquired, lungs had to be isolated from the surrounding body by manually drawn regions of interest (ROI) and in each CT slice, intraparenchymal densities and lung volume were measured. Total lung volume calculations were based on both gas-filled lung regions and intraparenchymal opacities and were calculated as sum of the lung volume of all CT slices. Whole intraparenchymal densities (including pulmonary blood volume and volume of the interstitium) were calculated as the volume-weighted mean of the ROI measurements of all CT slices.

\section{Statistical analyses}

Results are expressed as the median and 25th and 75th percentiles in order to give an indication of the sample distribution. Data are presented as measured during baseline (prelavage, not used for statistical comparison), after induction of ALI (postlavage) and during pc-CMV with incremental levels of PEEP $(0.5,1.0$ and $1.5 \mathrm{kPa})$, followed by PLV at the same levels of PEEP $(0.5,1.0$ and $1.5 \mathrm{kPa}$ ). Statistical analyses were performed using the SPSS for Windows software package (release 6.0, SPSS Inc., Chicago, IL, USA). Multivariate analyses of variance (MANOVA) for repeated measures was used. The statistical design for the MANOVA procedure was based on two within-factors, i.e. PEEP and PFC (indicating instillation of FC 3280), with PEEP having three and PFC having two levels. The levels of PEEP were $0.5,1.0$, and $1.5 \mathrm{kPa}$ during both ALI and PLV. The levels of PFC were ALI (prior to instillation of FC 3280) and PLV. To analyse further the effect of $\mathrm{CO}$ on $P \mathrm{a}, \mathrm{O}_{2}$ and $Q \mathrm{~s} / Q \mathrm{t}$ measurements, MANOVA was performed using $\mathrm{CO}$ as a time-dependent covariant. A p-value ð0.05 was considered significant. 
Table 1. - Haemodynamic data during the protocol

\begin{tabular}{|c|c|c|c|c|c|c|c|c|c|}
\hline & & PEEP $\mathrm{kPa}$ & & aseline & & ALI & & PLV & MANOVA \\
\hline \multirow[t]{3}{*}{$f \mathrm{C}$} & $\min ^{-1}$ & 0.5 & 84 & $(70,117)$ & 89 & $(81,137)$ & 138 & $(125,166)$ & \\
\hline & & 1.0 & & & 109 & $(71,145)$ & 145 & $(134,179)$ & \\
\hline & & 1.5 & & & 99 & $(72,143)$ & 142 & $(122,181)$ & \\
\hline \multirow{3}{*}{ MAP } & $\mathrm{mmHg}$ & 0.5 & 84 & $(72,113)$ & 93 & $(84,106)$ & 103 & $(80,121)$ & \\
\hline & & 1.0 & & & 90 & $(80,137)$ & 96 & $(81,110)$ & \\
\hline & & 1.5 & & & 92 & $(74,127)$ & 101 & $(72,117)$ & \\
\hline \multirow[t]{3}{*}{ PAP } & $\mathrm{mmHg}$ & 0.5 & 20 & $(14,22)$ & 27 & $(23,33)$ & 38 & $(31,42)$ & \\
\hline & & 1.0 & & & 30 & $(23,38)$ & 34 & $(32,41)$ & \\
\hline & & 1.5 & & & 31 & $(28,37)$ & 37 & $(31,38)$ & \\
\hline \multirow[t]{3}{*}{ CVP } & $\mathrm{mmHg}$ & 0.5 & 8 & $(5,13)$ & 8 & $(4,10)$ & 7 & $(5,12)$ & \\
\hline & & 1.0 & & & 6 & $(4,11)$ & 7 & $(6,12)$ & \\
\hline & & 1.5 & & & 8 & $(7,11)$ & 9 & $(5,12)$ & \\
\hline \multirow[t]{3}{*}{ PCWP } & $\mathrm{mmHg}$ & 0.5 & 11 & $(5,12)$ & 11 & $(7,13)$ & 5 & $(5,12)$ & \\
\hline & & 1.0 & & & 10 & $(6,13)$ & 6 & $(6,12)$ & \\
\hline & & 1.5 & & & 8 & $(6,12)$ & 8 & $(6,13)$ & \\
\hline \multirow[t]{3}{*}{$\mathrm{CO}$} & $\mathrm{L} \cdot \mathrm{min}^{-1}$ & 0.5 & 3.7 & $(3.5,5.3)$ & 4.4 & $(2.9,5.7)$ & 5.5 & $(4.6,6.8)$ & PEEP*, $p=0.04$ \\
\hline & & 1.0 & & & 3.7 & $(3.0,6.2)$ & 5.5 & $(4.3,6.4)$ & \\
\hline & & 1.5 & & & 3.7 & $(2.5,5.4)$ & 5.2 & $(3.7,6.3)$ & \\
\hline \multirow[t]{3}{*}{ SVR } & dyn $\mathrm{s} \cdot \mathrm{cm}^{-5}$ & 0.5 & 1398 & $(1132,2150)$ & 1775 & $(1181,2491)$ & 995 & $(772,2134)$ & \\
\hline & & 1.0 & & & 2197 & $(1203,2770)$ & 1123 & $(850,1981)$ & \\
\hline & & 1.5 & & & 2338 & $(1517,2590)$ & 947 & $(822,2526)$ & \\
\hline \multirow[t]{3}{*}{ PVR } & dyn $\mathrm{s} \cdot \mathrm{cm}^{-5}$ & 0.5 & 174 & $(149,192)$ & 276 & $(205,500)$ & 431 & $(213,579)$ & \\
\hline & & 1.0 & & & 499 & $(236,550)$ & 386 & $(247,628)$ & \\
\hline & & 1.5 & & & 584 & $(374,628)$ & 409 & $(236,550)$ & PEEP/PFC*, $\mathrm{p}=0.03$ \\
\hline \multirow[t]{3}{*}{$\mathrm{DO}_{2}$} & $\mathrm{~mL} \cdot \mathrm{min}^{-1}$ & 0.5 & 525 & $(426,627)$ & 280 & $(236,459)$ & 571 & $(381,914)$ & \\
\hline & & 1.0 & & & 378 & $(282,591)$ & 464 & $(446,859)$ & \\
\hline & & 1.5 & & & 478 & $(252,544)$ & 604 & $(447,864)$ & \\
\hline \multirow{3}{*}{$\mathrm{VO}_{2}$} & $\mathrm{~mL} \cdot \mathrm{min}^{-1}$ & 0.5 & 174 & $(97,187)$ & 168 & $(116,215)$ & 201 & $(72,263)$ & \\
\hline & & 1.0 & & & 173 & $(115,208)$ & 165 & $(129,205)$ & \\
\hline & & 1.5 & & & 181 & $(118,206)$ & 220 & $(150,247)$ & \\
\hline
\end{tabular}

Data are medians (and 25th, 75th percentile). Baseline indicates parameters prior to lung lavage (not used for statistical comparison); ALI: acute lung injury; PLV: partial liquid ventilation; MANOVA: multivariate analysis of variance. *: H0 hypothesis of equality of means of the measured data for variations of the respective interventions is rejected on a level of significance of 0.05 (PEEP*: significant effect of PEEP; $\mathrm{PFC}^{*}$ : significant effect of FC 3280; PEEP/PFC*: significant effect of combined application and p-values). $f \mathrm{C}$ : cardiac frequency; MAP: mean arterial pressure; PAP: mean pulmonary artery pressure; CVP: central venous pressure; PCWP: pulmonary capillary wedge pressure; CO: cardiac output; SVR: systemic vascular resistance; PVR: pulmonary vascular resistance; $\mathrm{DO}_{2}$ : oxygen delivery; $\mathrm{VO}_{2}$ : oxygen uptake. 1 $\mathrm{mmHg}=0.133 \mathrm{kPa}$.

Table 2. - Summary of gas exchange, respiratory mechanics and radiographical data

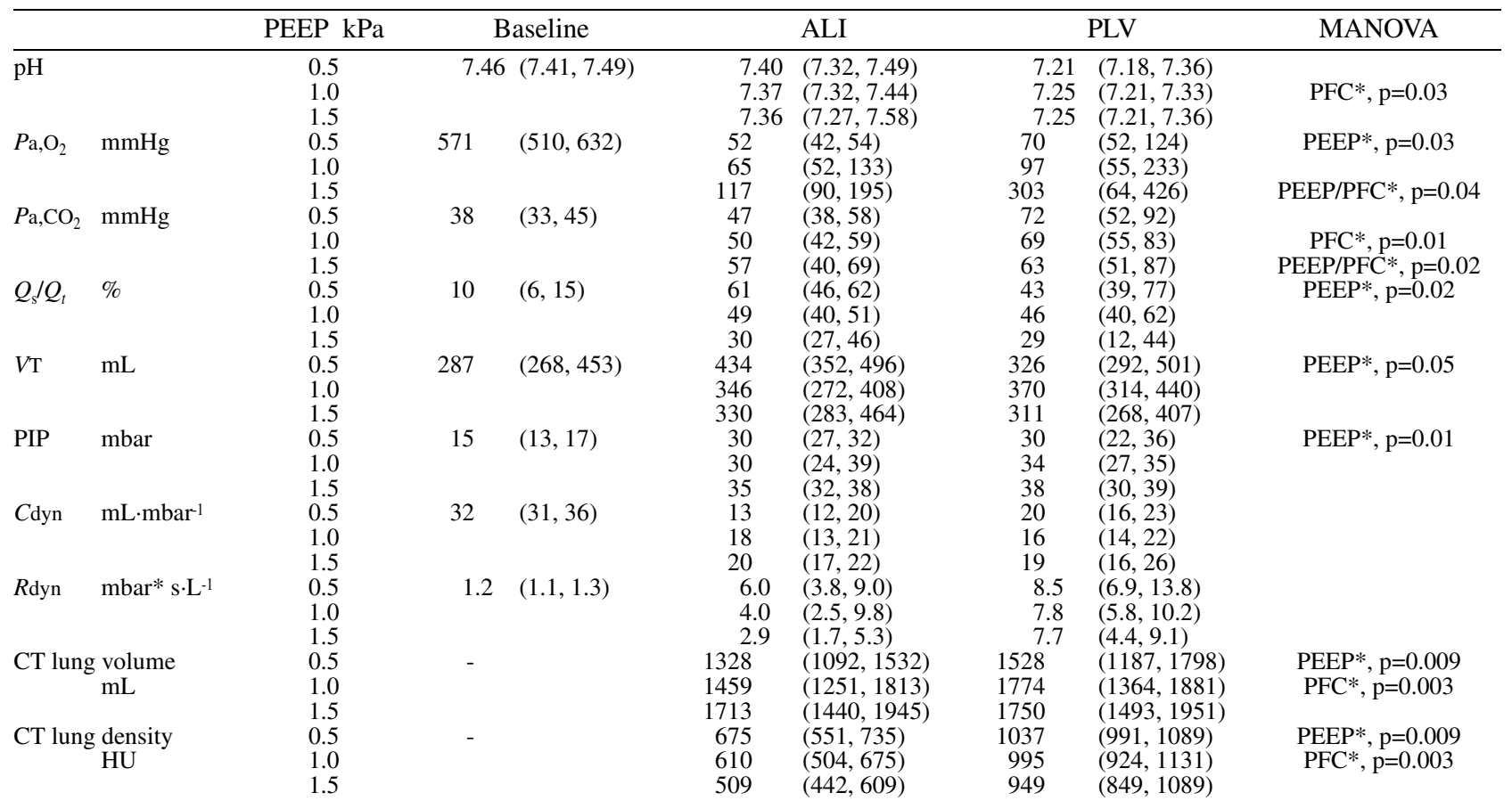

Data are medians (and 25th, 75th percentile). Baseline indicates parameters prior to lung lavage (not used for statistical comparison). ALI: acute lung injury; PLV: partial liquid ventilation; MANOVA: multivariate analysis of variance. *: H0 hypothesis of equality of means of the measured data for variations of the respective interventions is rejected on a level of significance of 0.05 (PEEP*: significant effect of PEEP; PFC*: significant effect of FC 3280; PEEP/PFC*: significant effect of combined application and $\mathrm{p}^{*} \mathrm{values}$ ). $P \mathrm{a}, \mathrm{O}_{2}:$ arterial oxygen tension; $P \mathrm{a}, \mathrm{CO}_{2}$ : arterial carbon dioxide tension; $Q_{s} / Q_{t}$ : pulmonary right-to-left shunt, $V \mathrm{~T}$ : tidal volume; PIP: peak inspiratory pressure; $C$ dyn: dynamic compliance; Rdyn: dynamic resistance; CT: computed tomography; $10 \mathrm{HU}$ : Houndsfield units. $1 \mathrm{mmHg}=0.133 \mathrm{kPa} ; 1 \mathrm{mbar}=0.1 \mathrm{kPa}$. 


\section{Results}

Prior to lung lavage, animals were comparable with regard to body weight and prestudy conditions. Following induction of ALI, PAP, $Q_{\mathrm{s}} / Q \mathrm{t}$ and $P \mathrm{a}, \mathrm{CO}_{2}$ were increased (tables 1 and 2). A marked decrease in $\mathrm{Pa}, \mathrm{O}_{2}$ and dynamic compliance $(C$ dyn) was observed in all animals, whereas dynamic resistance ( $R$ dyn) was increased (table 2 ).

In ALI and before PLV, application of PEEP induced a significant decrease in $\mathrm{CO}$ and $Q \mathrm{~s} / Q \mathrm{t} . P \mathrm{a}, \mathrm{O}_{2}$ was increased (tables 1 and 2). PEEP increased peak inspiratory pressure $(\mathrm{PIP})$ and decreased tidal volumes $(V \mathrm{~T})(\mathrm{p}<0.05)($ table 2$)$. $C$ dyn was increased and $R$ dyn decreased by administration of PEEP (NS).

Initiation of PLV significantly increased $P \mathrm{a}, \mathrm{CO}_{2}$ and decreased $\mathrm{pH}$. There was a tendency for $R$ dyn to increase following PLV (NS). Superimposing PEEP on PLV induced a significant increase in $\mathrm{Pa}, \mathrm{O}_{2}$, and a reduction in $\mathrm{Pa}_{\mathrm{a}}, \mathrm{CO}_{2}$ and pulmonary vascular resistance $(\mathrm{PVR})(\mathrm{p}<0.05)$. $C$ dyn and $R$ dyn were unchanged (tables 1 and 2).
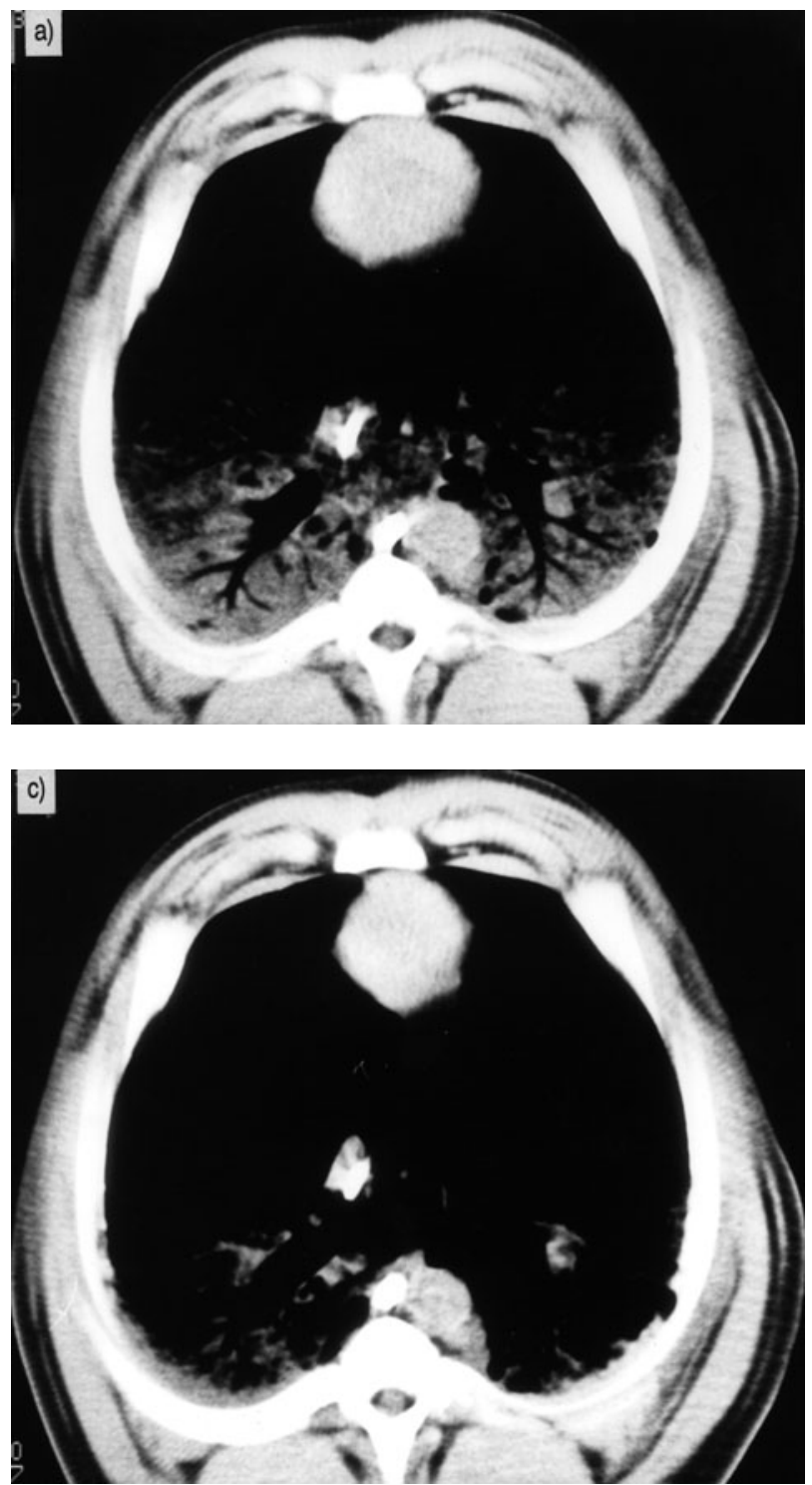

To analyse further the effect of $\mathrm{CO}$ on $Q_{\mathrm{s}} / Q_{\mathrm{t}}$ and $P \mathrm{a}, \mathrm{O}_{2}$ measurements during the study, MANOVA was performed using $\mathrm{CO}$ as a time-dependent covariant, demonstrating a significant effect of $\mathrm{CO}$ on $Q_{\mathrm{s}} / Q_{\mathrm{t}}$ during pc-CMV with PEEP $(\mathrm{p}<0.05)$. No statistical significant dependence of $\mathrm{Pa}, \mathrm{O}_{2}$ values on $\mathrm{CO}$ could be verified.

After lavage, CT scans of the lungs revealed a patchwork of normal and dense areas with generally well-defined boundaries. In all animals, dense areas of variable size were visible in the dependent lung regions (fig. 1a). In ALI and before PLV, application of PEEP resulted in a significant decrease in intraparenchymal lung density. Lung volume was significantly increased (table 2 , fig. $1 \mathrm{~b}, \mathrm{c}$ ).

The radiodensity of FC 3280 was determined at 553 Houndsfield units (HU). Initiation of PLV induced a significant increase in both CT lung volume and intraparenchymal densities (table 2), with PFC predominantly pooling in dependent lung regions creating a vertical gradient in density (fig. 2a). Administration of PEEP during PLV induced a spreading of PFC from the tracheobronchial system into the lung periphery, e.g. the bronchial system and the alveoli (fig. 2b, c).

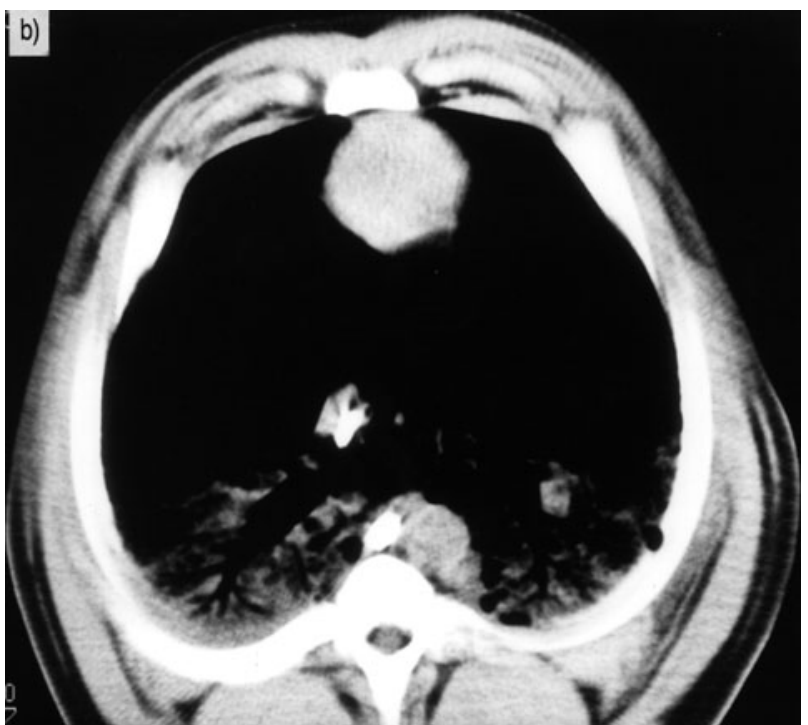

Fig, 1. - a) Cosmputed tomegraply (CT) of the lung folkowine indaction of acute lung injury (ALI) (pressure-controlled mechanical ventilation (Fe-CMVh positive end-espiratory pressure (PEEP) D.5 kPa). Tuble position is at the level of the lower lobes, $4 \mathrm{~cm}$ below the carina. Scans

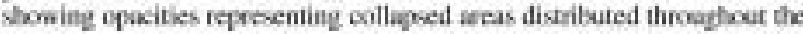
lungs in a nonhomogeneous manner, bot predominantly concentrating in dependent (posterios) lung regions. Note the air bronchograms in the dependent and in the middle regions of the lung. b) CT of the lung following induction of ALII (p)-CMV, PEEP $1.0 \mathrm{kPa}$ ) showitg an increase in the air content of the lung. Note a roduction in consolidations in the dependent focnterion) lung with increasing kevels of PEFP. c) CT of the lung following induction of ALT (pc-CMV, PEEP $1.5 \mathrm{kPa}$ ) showing a furiber increase in the air concem of the lung. Opacities representing collapeed areas in the dependent (posterion) lung have almost disappoared. 

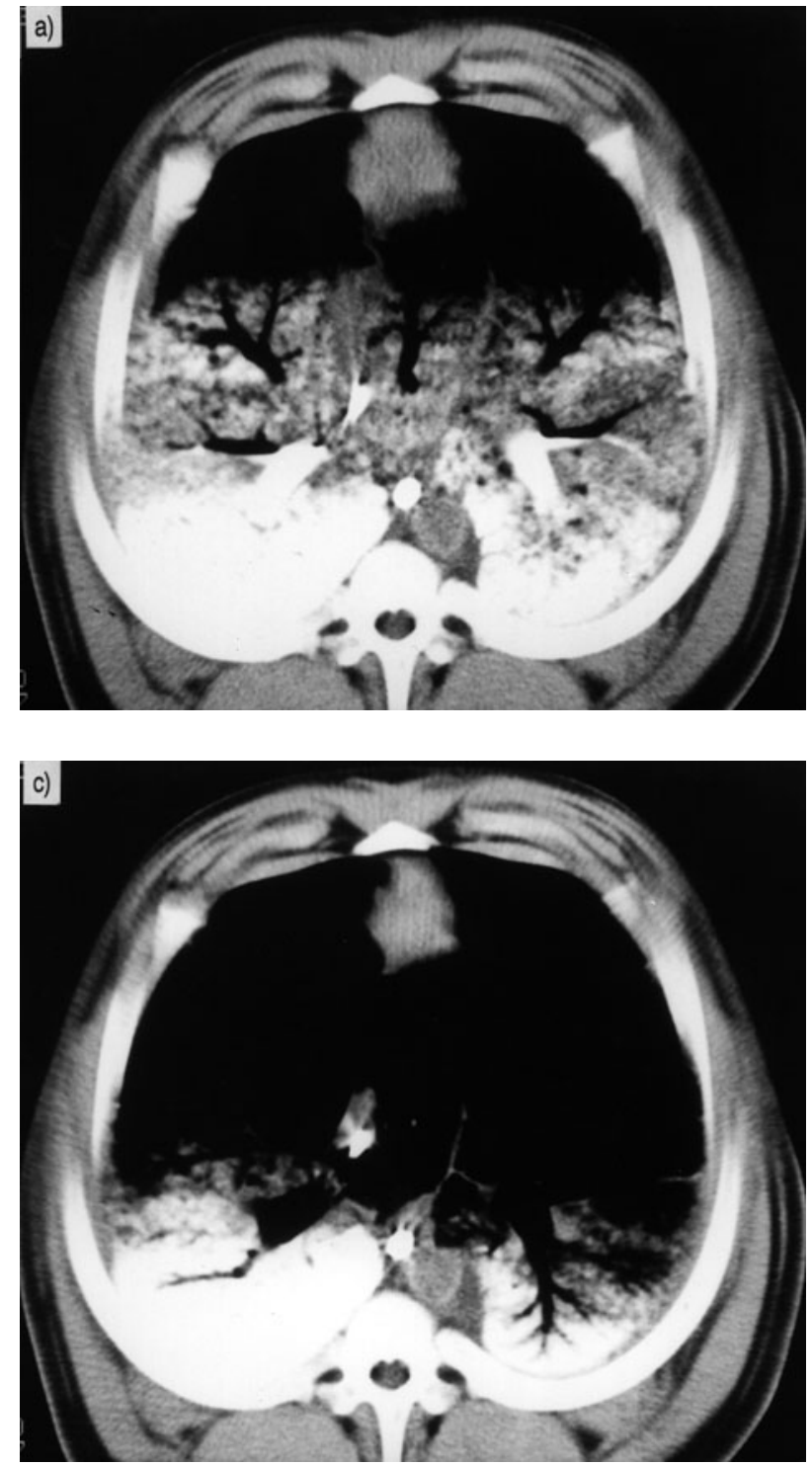

\section{Discussion}

In the present study, CT of the acutely injured lung revealed a significant increase in lung volume and a reduction in lung density by PEEP, presumably as an equivalent of alveolar expansion. PLV alone significantly increased CT lung volume and lung density. Coapplication of incremental levels of PEEP during PLV was highly effective in terms of oxygenation, although neither a further increase in CT lung volume nor a significant reduction in lung density was demonstrated. Radiographically, a distribution of PFC mainly into dependent lung regions was confirmed and the administration of PEEP during PLV induced a spreading of PFC from the tracheobronchial system into the lung periphery, e.g. into the bronchial system and the alveoli. It should be pointed out that lung volume calculations by CT included lung interstitium and pulmonary blood volume. This reduced the accuracy of CT lung volume calculations since PLV and the different levels of PEEP applied may have altered the intrapulmonary blood volume. Furthermore, alveolar expansion could not be dis-

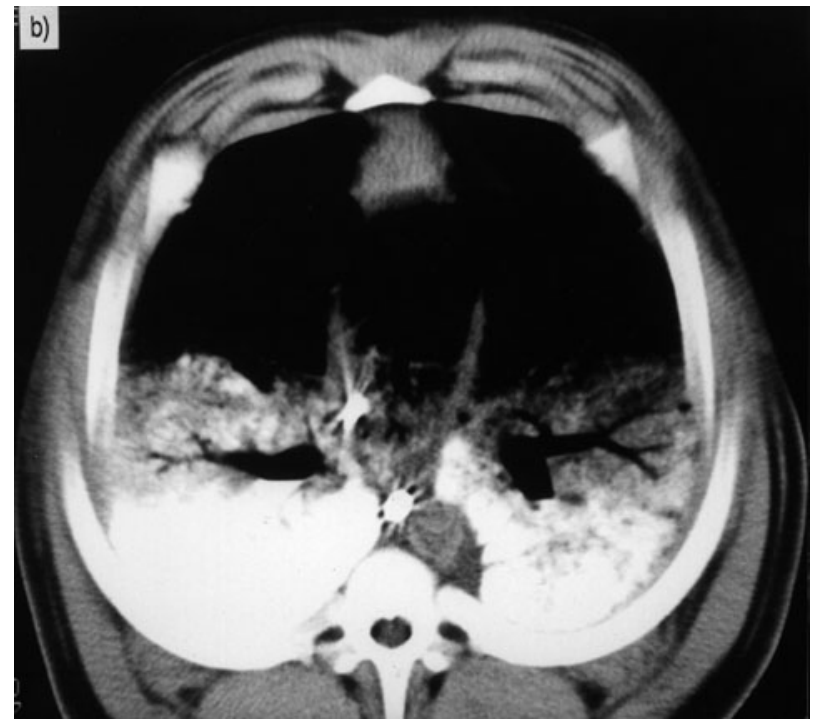

Fig. 2. - a) Congened womography (CT) of the lung following purtial liquid ventilation (PLV) with $30 \mathrm{~mL} \cdot \mathrm{kg}^{-1} \mathrm{FC} 3280$ (pressure-controlled mechanical ventilicion (je-CMV), positive end-eypinacey pessure (P'F.P') $0.5 \mathrm{kPa}$ ). Table position as in fig. I. The CT scans show the nonhemsoeneous distriburion of the highy matiopacae perflusencartoms $(\mathrm{PFC})$ is both Jungs. Opacitics, predominantly in the posterior lung, represent a combination of PFC and ockemalavage fluid remaining in the airways, erentually flosking on fro of the senve PFC and filling alveoli above the PFC-filled regions of the lung. The larger airways of the posterior lung are completely filled with PFC, whereas air bronchograms are shown in the middle regisns of the lung, b) CT wan of the lung following PLV with $30 \mathrm{~mL} \cdot \mathrm{kg} / \mathrm{FC}$ (pc-CMV, PEEP $10 \mathrm{kPa}$ ) demonstrating a revuction in opacities with a trore solidly opaque portion in the right posterion lung. In larper airways, small levels of PFC can be seen. c) CT scan of the lung following PL.V with $30 \mathrm{~mL}-\mathrm{kg}-1 \mathrm{FC}$ 3281) (pe-CMV, PEEP I.5 $\mathrm{kPa}$ ) showing a further reduction in opacities and a more solidly opaque portios of PFC is the right posterior, and less in the left posterice lung with air bronchograms. The amount of intrapulmenary PFC liquid appears to be decreased as a result of distribution from the tracheobronchial system into the lung periphery, e.g. the bronchial system and the alveoli.

criminated from compression of atelectatic lung regions by overexpansion of adjacent ventilated lung areas. In a preliminary report, GoLDNER et al. [10] recently demonstrated that in healthy lungs, stepwise instillation of doses of PFC, each equalling one-third of FRC, induced a dosedependent decrease in aerated lung volume. Total FRC (gas-filled and PFC-filled, determined by helium dilution), however, was increased at higher doses of PFC (approximating two-thirds of FRC). The authors speculated that PLV associated changes in transpulmonary pressure might induce volume loss after the first dosing of PFC.

In ARDS, it has been shown that PEEP-induced changes in CT intraparenchymal opacities were well correlated with changes in oxygenation [11]. During PLV (with an $F \mathrm{I}_{2} \mathrm{O}_{2}$ of 1.0 ) the concentration of $\mathrm{O}_{2}$ in PFC-filled alveoli is reduced compared with that in gas-ventilated alveoli (FC 3280 dissolves a maximum of $40 \mathrm{vol} . \%$ of $\mathrm{O}_{2}$, compared with 100 vol.\% present in pure oxygen gas). Thus, it has been suggested that the beneficial effects of PLV on gas exchange are attributed to the filling, distending and stabilization of alveoli with the noncompressible PFC, 
thereby reopening air spaces and preventing them from end-expiratory collapse, an effect similar to that of PEEP [4]. In ALI, this might be particularly true in dependent zones of the lung, where the expanded weight of the infiltrated lung causes airway closure and alveolar collapse along the vertical axis of the thorax, concentrating compression atelectasis in these dependent regions [2]. In PLV, the same gravitational force providing recruitment of dependent alveoli may, furthermore, redirect pulmonary blood flow towards nondependent, ventilated lung regions [12]. Improved homogeneity of $V^{\prime} \mathrm{A} / Q^{\prime}$ resulting from the recruitment of alveoli and redirection of blood flow should therefore mainly account for improvements in oxygenation during PLV.

In the present study, however, application of PEEP alone was more effective than PLV alone, significantly increasing $\mathrm{Pa}, \mathrm{O}_{2}$ along with an increase in CT lung volume, indicating recruitment due to a reduction of collapsed alveoli with zero ventilation/perfusion ratio $\left(V^{\prime} \mathrm{A} / Q^{\prime}\right)$. Superimposing PEEP on PLV significantly increased pulmonary gas exchange, probably through an improved matching of $V^{\prime} \mathrm{A} / Q^{\prime}$. The high spreading coefficients and low surface tensions of PFC produce surfactant-like activity by reducing surface tension at air liquid interfaces. In ALI, PFC maintain low, but constant air PFC surface tensions for any given alveolar radius. If the alveolus is not completely filled with PFC, end-expiratory alveolar stability at low end-expiratory volumes (low FRC) could not be presumed and end-expiratory collapse may occur. It may be speculated that during PLV in ALI three different compartments might be separated within the lung: 1) a PFCfilled compartment in the dependent parts of the lung; 2) an intermediate compartment where alveoli are partially PFC-filled; and 3) a non-PFC-filled compartment in nondependent lung regions. In the latter ones, alveolar stability at low end-expiratory volumes is dependent on a functioning endogenous surfactant system [13] and application of high enough levels of PEEP will prevent end-expiratory collapse. The data indicate that in ALI, despite high-dose PLV (30 $\left.\mathrm{mL} \cdot \mathrm{kg}^{-1}\right)$, significant $V^{\prime} \mathrm{A} / Q^{\prime}$ mismatching occurs and higher levels of PEEP are essential to provide appropriate end-expiratory lung volume. This hypothesis is partly supported by a previous study by SALMAN et al. [14], demonstrating alveolar collapse during weaning back from PLV (i.e. at low volumes of PFC) if insufficient levels of PEEP were applied.

HerNAn et al. [5] assessed the cumulative effects of PEEP levels between $0.6-1.4 \mathrm{kPa}$ at a constant tidal volume during PLV as insignificant for pulmonary gas ex-change in healthy sheep. In their experiments the authors defined end-inspiratory pressure and tidal volume as de-terminants of oxygenation. In normal sheep undergoing PLV, FRC might already be recruited during mechanical ventilation with a PEEP of $0.6 \mathrm{kPa}$ and application of higher levels of PEEP will cause overexpansion of alveoli. Nevertheless, after initiation of PLV, HeRNAN et al. [5] described PEEP as a means to clear proximal airways of PFC, thus preventing pressure-spikes during gas inflation. In their study, a second group of adult sheep $(58 \pm 8 \mathrm{~kg})$ with hydrochloric acid-induced ALI was enrolled, receiving volume-controlled CMV (PEEP $1.0 \mathrm{kPa}$, PIP $6.2 \pm 0.8 \mathrm{kPa}$ ) resulting in a $P \mathrm{a}, \mathrm{O}_{2}$ of $13.3 \mathrm{kPa}(<100 \mathrm{mmHg})$ before randomization. PLV significantly improved oxygenation, but the effects of incremental levels of PEEP during PLV were not investigated in this group of sheep.

In the present protocol, application of incremental levels of PEEP in ALI significantly impaired CO, probably mainly owing to an increase in intrathoracic pressure and a reduction in venous return. PEEP-induced circulatory effects are, however, considered to depend on many factors, including lung and chest-wall compliance, ventricular loading conditions, contractility and the sympathoadrenergic compensatory response [15]. During PLV and PLV/PEEP, CO tended to be higher than during ALI, however, the difference was not significant. To analyse further the effects of $\mathrm{CO}$ on $Q_{\mathrm{s}} / Q \mathrm{t}$, MANOVA was performed using $\mathrm{CO}$ as a time-dependent covariant. During pc-CMV with PEEP a significant effect of $\mathrm{CO}$ on $Q_{\mathrm{s}} / Q_{\mathrm{t}}$ was demonstrated. Previously, PEEP-induced depression of CO has been identified as a mechanism of shunt reduction in ALI. This was attributed to a decrease in perfusion of unventilated lung [16]. In the present study, the increase in CO during PLV was not associated with an increase in $Q_{\mathrm{s}} /$ $Q \mathrm{t}$, suggesting no increase in the fractional perfusion of units with low $V^{\prime} \mathrm{A} / Q^{\prime}$.

Initiation of PLV had no adverse effects on haemodynamics in this series. On the contrary, $\mathrm{CO}$ and consequently $\mathrm{DO}_{2}$ tended to be higher during PLV (NS), an observation previously reported in larger animals [5]. Coapplication of PEEP in PLV significantly decreased PVR, probably through a concomitant improvement in oxygenation and a reduction in $P \mathrm{a}, \mathrm{CO}_{2}$ which may have resulted in a decrease in hypoxic pulmonary vasoconstriction.

Initiation of PLV during pc-CMV (with an essentially unchanged $V \mathrm{~T}$ ) induced a significant increase in $\mathrm{Pa}_{\mathrm{a}} \mathrm{CO}_{2}$, suggesting an increase in alveolar dead space ventilation. Superimposing PEEP during PLV significantly reduced $P \mathrm{a}, \mathrm{CO}_{2}$, indicating an improvement in alveolar ventilation. Total liquid ventilation has been demonstrated potentially to reduce effective alveolar ventilation, thus impairing $\mathrm{CO}_{2}$ removal. This has been primarily attributed to diffusion limitation, caused by the "diffusion dead space" [17]. During PLV, however, $\mathrm{CO}_{2}$ removal might be effectively improved by increasing minute ventilation.

In the piglets in the present study, PIP was limited to 4.0 $\mathrm{kPa}$, although the pressures required to normoventilate the animals prior to ALI were much lower (table 2). This mode of ventilation resulted in tidal volumes of approximately $8-10 \mathrm{~mL} \cdot \mathrm{kg}^{-1}$ at baseline (healthy pigs). After induction of lung injury, PIP was increased to values $ð 4.0$ $\mathrm{kPa}$, resulting in $V \mathrm{~T}$ of up to $14 \mathrm{~mL} \cdot \mathrm{kg}^{-1}$; however, normoventilation was not accomplished and mild hypercapnia was accepted. High $V \mathrm{~T}$ has been shown potentially to induce volutrauma in ALI [18]. Alveolar overinflation, increased shear forces and finally alveolar disruption are consequences of volu-trauma. We did not evaluate ventilator-induced lung injury in this study; however, current concepts of mechanical ventilation in ALI propagate CMV at low airway pressures and low $V \mathrm{~T}$ [19].

In ALI, an increase in $R$ dyn has been attributed to an increase in the viscoelastic resistance of tissue and a reduction in accessible lung volume [20]. Initiation of PLV induced an additional increase in $R$ dyn, probably due to the fluid viscosity of PFC in small airways, using the first portion of airway pressure applied to move the dense liquid into the alveoli. Thus, the lung becomes functionally a slower compartment, clinically apparent as pressure-spike 
readings following the implementation of gas ventilation [5]. In our series the application of incremental levels of PEEP did not counterbalance the increase in $R$ dyn. The flow and volume data reported are considered to apply purely to the animals' respiratory mechanics, excluding the effects of additional resistance due to the ETT [7]. The accuracy of $P$ trach corrections for the ETT during PLV have, however, not been systematically evaluated and PFC mucus formation might have significantly affected the accuracy of $P$ trach corrections for the ETT.

In oleic acid-induced ALI, BARBAS et al. [21] demonstrated that PLV (with doses of PFC approximating FRC) induced a significant increase in regional (dependent) pleural pressure and an increase in the vertical pleural pressure gradient. Moreover, the authors showed that levels of PEEP up to $2.7 \mathrm{kPa}$ could decrease the vertical pleural pressure gradient, possibly owing to a more uniform distribution of alveolar gas. These preliminary data suggest that during PLV and during its combined use with PEEP, regional changes in pleural pressures could generate regional alterations in lung volumes and respiratory mechanics. Thus, calculations of compliance using standard pressure-volume measurements will not necessarily reflect the regional behaviour of lung mechanics. Further research is needed to specify regional alterations in transpulmonary pressure and surface tension and their effects on respiratory mechanics during PLV.

In conclusion, it was demonstrated that in experimental ALI, PEEP effectively provided alveolar recruitment and improved oxygenation. During PLV, incremental levels of PEEP provided a significant improvement in gas exchange and were haemodynamically well tolerated. It is tempting to speculate that the combined application of PLV and PEEP might eventually allow either a reduction in the volumes of PFC required or in the PEEP necessary to maintain pulmonary gas exchange in ALI.

Prior to its clinical usage in patients with acute respiratory distress syndrome, further research should help to clarify both the optimal dosing of perfluorocarbons and the appropriate ventilatory settings during partial liquid ventilation.

Acknowledgements: The authors would like to thank K. Kelly, M. Max, T. Ehrenstein, A. Quaack, V. Eichorn, U. Tschaktsch, A. Ullmann, M. Baum and D. Pahl for their excellent technical assistance.

\section{References}

1. Falke KJ, Pontoppidan H, Kumar A, Lith DE, Geffin B, Laver MB. Ventilation with end-expiratory pressure in acute lung disease. J Clin Invest 1972; 51: 2315-2323.

2. Gattinoni L, D'Andrea L, Pelosi P, Pesenti A, Fumagalli R. Regional effects and mechanism of positive end-expiratory pressure in early adult respiratory distress syndrome. JAMA 1993; 269: 2122-2127.

3. Fuhrman BP, Paczan PR, DeFrancis M. Perfluorocarbonassociated gas exchange. Crit Care Med 1991; 19: 712722.

4. Marini JJ. Down side up: a prone and partial liquid asset. Intensive Care Med 1995; 21: 963-965.
5. Hernan LJ, Fuhrman BP, Kaiser RE, et al. Perfluorocarbon-associated gas exchange in normal and acid-injured large sheep. Crit Care Med 1996; 24: 475-481.

6. Mates EA, Hildebrandt J, Jackson JC, Tarczy-Hornoch P, Hlastala MP. Shunt and ventilation-perfusion distribution during partial liquid ventilation in healthy piglets. $J$ Appl Physiol 1997; 82: 933-942.

7. Guttmann J, Eberhard L, Wolff G, Bertschmann W, Zeravik J, Adolph M. Maneuver-free determination of compliance and resistance in ventilated ARDS patients. Chest 1992; 102: 1235-1242.

8. Guttmann J, Eberhard L, Fabry B, Bertschmann W, Wolff G. Continuous calculation of intratracheal pressure in tracheally intubated patients. Anesthesiology 1993; 79: 503513.

9. Lachmann B, Robertson B, Vogel J. In vivo lung lavage as an experimental model of the respiratory distress syndrome. Acta Anaesthesiol Scand 1980; 24: 231-236.

10. Goldner M, Shapiro R, Amato M, et al. Effect of incremental perfluorocarbon instillation on lung volumes and regional pleural pressures (Abstract). Am J Respir Crit Care Med 1997; 155: A87.

11. Gattinoni L, Mascheroni D, Torresin A, et al. Morphological response to positive end expiratory pressure in acute respiratory failure: computerized tomography study. Intensive Care Med 1986; 12: 137-142.

12. Shaffer TH, Lowe CA, Bhutani VK, Douglas PR. Liquid ventilation: effects on pulmonary function in distressed meconium-stained lambs. Pediatr Res 1984; 18: 47-52.

13. Taskar V, John J, Evander E, Robertson B, Jonson B. Surfactant dysfunction makes the lungs vulnerable to repetitive collapse and reexpansion. Am J Respir Crit Care Med 1997; 155: 313-320.

14. Salman NH, Fuhrman BP, Steinhorn DM, et al. Prolonged studies of perfluorocarbon associated gas exchange and the resumption of conventional mechanical ventilation. Crit Care Med 1995; 23: 919-924.

15. Pinsky MR. The hemodynamic consequences of mechanical ventilation: an evolving story. Intensive Care Med 1997; 23: 493-503.

16. Dantzker DR, Brook CJ, Dehart P, Lynch JP, Weg JG. Ventilation-perfusion distributions in the adult respiratory distress syndrome. Am Rev Respir Dis 1979; 120: 10391052.

17. Koen PA, Wolfson MR, Shaffer TH. Fluorocarbon ventilation: maximal expiratory flows and $\mathrm{CO}_{2}$ elimination. Pediatr Res 1988; 24: 291-296.

18. Dreyfuss D, Soler P, Basset G, Saumon G. High inflation pressure pulmonary edema: respective effects of high airway pressure, high tidal volume and PEEP. Am Rev Respir Dis 1988; 137: 1159-1164.

19. Muscedere JG, Mullen JBM, Slutsky AS. Tidal ventilation at low airway pressures can augment lung injury. $A m$ Rev Respir Dis 1994; 149: 1327-1334.

20. Tantucci C, Corbeil C, Chassé M, et al. Flow and volume dependency of respiratory system flow resistance in patients with the adult respiratory distress syndome. Am Rev Respir Dis 1992; 145: 355-360.

21. Barbas CSV, Amato MBP, Goldner M, et al. Effect of PEEP on regional pleural pressures during partial liquid ventilation in dogs with oleic acid induced lung injury (Abstract). Am J Respir Crit Care Med 1997; 155: A86. 